\title{
ARTICLE \\ Discussion on the Construction of Ecological Water Network in Guangxi Province of China
}

\author{
Jinhua Shao ${ }^{1,2,3}$ Kai Huang ${ }^{2,3}$ Wei Tang ${ }^{2,3}$ Xinyue Liang ${ }^{2,3}$ Weixiong Wu ${ }^{2,3}$ Guoqin Huang $^{1^{*}}$ \\ 1. Research Centre on Ecological Sciences, Jiangxi Agricultural University, Nanchang, Jiangxi, 330045, China \\ 2. Guangxi Hydraulic Research Institute, Nanning, Guangxi, 530023, China \\ 3. Guangxi Key Laboratory of Water Engineering Materials and Structures, Nanning, Guangxi, 530023, China
}

\section{ARTICLE INFO}

Article history

Received: 21 June 2021

Accepted: 20 July 2021

Published Online: 19 August 2021

\section{Keywords:}

Water engineering

Management system

Publicity and education

\section{Introduction}

In order to pursue the rapid increase of economy and a higher working efficiency, many ecological and environmental problems were ignored in the past ${ }^{[1]}$,especially ecological water problem. Water pollution, soil erosion and deterioration of ecological environment in some areas have become increasingly prominent. In Chinese main river basins (Yellow River, Songhua River, Huai River and so on), scientists found that the ammonia nitrogen, COD (chemical oxygen demand) and other measured index were above standard value. The same situation happened in the Three Gorges Reservoir area, the amount of heavy metals and pollutant exceeding the normal standard. The influence of soil erosion in China is in a wide range. The Loess Plateau is the most serious area that suffered from soil erosion, according to the observation, the Yellow River sediment

\section{ABSTRACT}

The water network plays an important role in maintaining the stability of regional water resource and ecological environment. It is also affecting the harmonious development between environment and economy. Guangxi is one of the provinces with relatively rich water resources in China, while the ecological water network exists deficiencies and faces challenges. The current situation and defects of ecological water network in Guangxi province will be discussed. By studying the experience of the establishing and the preserve of ecological water network in various regions at home and abroad, some suggestions and targeted measures will be mentioned for a better ecological water network in Guangxi.

transport is about 1.6 billion tons per year, $90 \%$ of which comes from the Loess Plateau. In order to address ecological water problem systematically and enhance the water security ability comprehensively. It is necessary to improve the regional water environment, strengthen the water ecological restoration ability and developing water landscape. Not only the rapid economic growth, but also the ecological water networks should be considered. Constructing ecological water network system have become one of the significant contents of water conservancy development in the fourteenth five- year plan.

\section{The Development Status Domestic and Abroad}

\subsection{The Water Network Development}

In the $21^{\text {st }}$ century, Shandong province is one of the

*Corresponding Author:

Guoqin Huang

Research Centre on Ecological Sciences, Jiangxi Agricultural University, Nanchang, Jiangxi, 330045, China;

Email:hgqjxes@sina.com 
earliest provinces to conduct the construction of modern water network in China. Liu Yongyi and Wang Weiping ${ }^{[3]}$ studied the operation law of modern water network through the provincial, municipal and county network architecture water network system. The management of modern water network should be unified by various level water administration, and administrative departments maintain communication to establish a regional water network. With the proposing of digital river basin research and the successfully demonstrating of digital river basin application systems such as Digital Yellow River ${ }^{[4]}$ and Digital Qingjiang River ${ }^{[5]}$, the research of digital water network has been gradually carried forward. In addition, the relevant information of water network was extracted digitally ${ }^{[6]}$ by $3 \mathrm{~S}$, visual simulation, communication engineering, unmanned aerial vehicle (UAV) and other advanced technologies to construct the digital water network system. In 2004, the concept of "intelligent water network" was put forward firstly in Australia ${ }^{[7]}$. The network offers an internet platform to different regional departments. Based on geographic information, departments can exchange latest hydraulic information, then the related data were processed and integrated for a better use. In addition, the intelligent water network can exhibit technological process, for example, seawater desalination. In 2013, China national intelligent water network ${ }^{[8]}$ project framework was formed preliminarily, leading to the digital water network has been further upgraded to the level of digital water network and intelligent water network. Intelligent water network ${ }^{[9]}$ is such an integrated management and control platform that comprises water physics, water information and water management through intelligent means and technologies. And water network construction has entered a more intelligent era since then.

\subsection{The Water Ecological Development}

The ecological oriented optimal allocation of water resources ${ }^{[10]}$ has always been a hot topic discussed by experts domestic and abroad. In 2020, Li et al have showed, the ecological water network can guarantee the water area, ecological corridor and ecological diversity better than the independent river system, once the original natural habitat and ecological process of the ecological water network are destroyed, it will not only threaten the regional ecological security, but also gradually lose the regional landscape features ${ }^{[11]}$. If fish migration areas or foraging areas have been damaged, the survival of fish stocks will be threatened. Britain ${ }^{[12]}$ has developed a relatively perfect legal system for this aspect. As early as in The Water Resources Act of 1963, there was a regulation on "minimum acceptable flow". As early as 2005, in China, Hubei province ${ }^{[13]}$ issued a variety of laws and regulations on water environment and water ecological protection in order to vigorously promote the construction of water ecosystem. However, the construction of water ecological network has been advancing quite slowly ${ }^{[14]}$.

\section{Current Situation and Existing Problems of Water Network Construction in Guangxi Province}

\subsection{Current Situation of Water Ecological Resources}

Guangxi is one of the provinces with rich water resources in China ${ }^{[15]}$, with developed water system and numerous rivers. The whole region ${ }^{[16]}$ belongs to four river basin of Pearl River Basin, Yangtze River Basin, coastal basin and Red River Basin, accounting for $85.4 \%, 3.5 \%, 10.3 \%$ and $0.8 \%$ respectively. There are 1350 rivers with water-collecting area ${ }^{[17]}$ of $50 \mathrm{~km}^{2}$ and above, with a total length of $52386 \mathrm{~km}$. There are more than 4550 reservoir projects and more than 40000 dams in the region. Meanwhile, Guangxi is also one of the three provinces with the richest biodiversity in China ${ }^{[18]}$. There are 8562 species of wild plants ranking the third in China and 1149 species of terrestrial vertebrate wildlife, ranking second in China, while fish account for nearly a quarter of the total freshwater fish species in China. The area of contiguous wetlands is 754300 hectares, and forest ${ }^{[19]}$ covers an area of 14.8 million hectares, with a forest coverage rate of $62.37 \%$ ranking third in China. 78 nature reserves have been built ${ }^{[20]}$, occupying $5.3 \%$ of the total land area of the region. Consequently, there are $90 \%$ of the national key protected wild animal and $82 \%$ of the national key protected botanic species in Guangxi, as well as $31 \%$ of mangrove wetlands.

\subsection{Main Problems}

(1) Insufficient connection water system of rivers, lakes and reservoirs

The first problem is that the serious shrinkage of the flow capacity of rivers and insufficient connectivity. In Guangxi, due to the long-term lack of maintenance and fix, some of the water conservancy infrastructures such as rivers, lakes are damaged and broken. Besides, some villagers exploit and utilize water resources unreasonably and other fill clay into the river illegally for expanding land, which contribute to flow rate reduced and river siltation. At the same time, the self-purification ability of water ecology is reduced lading to the siltation of some rivers with a trend of aggravation ${ }^{[21]}$. These phenomena 
not only reduce the ability to resist flood disaster, but also seriously damage the well-organized development of water ecological environment.

(2) Serious damage of water ecological environment

In Guangxi, Almost all the rural sewage is directly discharged into the river, According to Guangxi Zhuang Autonomous Region Water Resources Bulletin 2013, In July 2013, the river reach of Hejiang River in Liantang Town and Butou Town, Babu District, Hezhou City suffered from unexplained death of cage fish culture. It was found that the water quality of Hejiang river reach exceeded the standard of Cadmium and Thallium.The domestic sewage and industrial waste water in some rural area in Guangxi ${ }^{[22]}$ were directly discharged into rivers without treatment, especially in the river sections flowing through cities, where the water quality was poor, and there was threaten in the safety of some drinking water sources. The existing of illegal fishing led to the decline of fishery resources and a serious threat to the survival of wild species. In addition, the disordered sand mining in the river has damaged the living environment of aquatic animals and plants. At the same time, the invasion of alien species has damaged the structure and function of water ecosystem, which has weakened the diversified characteristics of the river and caused serious harm to people's health and ecological environment. According to the survey, in rural rivers, the number of fish stocks in rivers in Guangxi is almost two third less than that in the 1980s, The number of black carp, grass carp, silver carp and Bighead Carp decreased significantly, and the weight of the fish was significantly lower than that of the 1980s.

(3) The management system of ecological water network is not perfect

In Guangxi, the management system of ecological water network exists drawbacks. First of all, the inadequate management of water conservancy infrastructure construction, including shoreline collapse, sediment deposition and overgrown weeds, which lead to the shrinkage of river water storage space and the loss of ecological function of river corridor. The frequent floods during the wet season, and the weakening or even exhaustion of river base flow during the dry season. Secondly, there was a lack of the ability of overall deployment and industry supervision of ecological water network. There was a lack of unified administrative management for water ecological problems, but with a phenomenon of multi management ${ }^{[24]}$. For example, for the same water ecological problem, the administrative authority was often dispersed to different departments such as water conservancy, agriculture, forestry, environmental protection, fishery, culture and tourism, and each department has not established a data sharing management platform, which was easy to cause unclear responsibilities, multiple politics, buck passing, information barrier and low efficiency. In addition, due to the lack of effective compensation mechanism for water ecological construction, the contradiction between water ecological protection and people's life and production was difficult to coordinate comprehensively, which obviously weakened the protection of water ecology. Therefore, the construction of ecological water network management system was required to be further improved in order to realize the efficient management of modern ecological water network.

(4) The concept of water ecological protection is relatively out of date

Compared with the leading areas of water ecological protection in China such as Jiangsu, Zhejiang ${ }^{[25]}$, the concept of water ecology in Guangxi was relatively out of date. First of all, the strength of water ecology publicity and education were not enough, so that the public doesn't have the awareness of the value and importance of water ecology. Secondly, there was a lack of regional cultural characteristics in the construction of water ecological scenic spots, so that the investment of social capital was not enough. At the same time, the financial investment of government departments was very limited, resulting in the lack of effective investment and financing mechanism in the construction of ecological water network, and the lack of public concern about the ecological environment protection. The government is more inclined to display propaganda slogans, but neglects to ask the professional team of water ecological protection to guide the public on how to protect the water ecological environment.

\section{Countermeasures of Ecological Water Network Construction in Guangxi Province}

Under the guidance of the Chinese water management policy of "giving priority to water saving, spatial balance, systematic management, and two pronged approach", combined with the overall situation of water resources development, utilization and industrial layout of regional economic development with Guangxi characteristics. Taking the integration of water resources, ecology, environment and economy as the construction goal, to build a new pattern of Guangxi ecological water network with complete system, coordinated functions, intensive and efficient development, green intelligence, wellorganized regulation, safety and reliability.

\subsection{Construction of Ecological Water Network with Interconnected Water Systems}

The related administrators and water management 


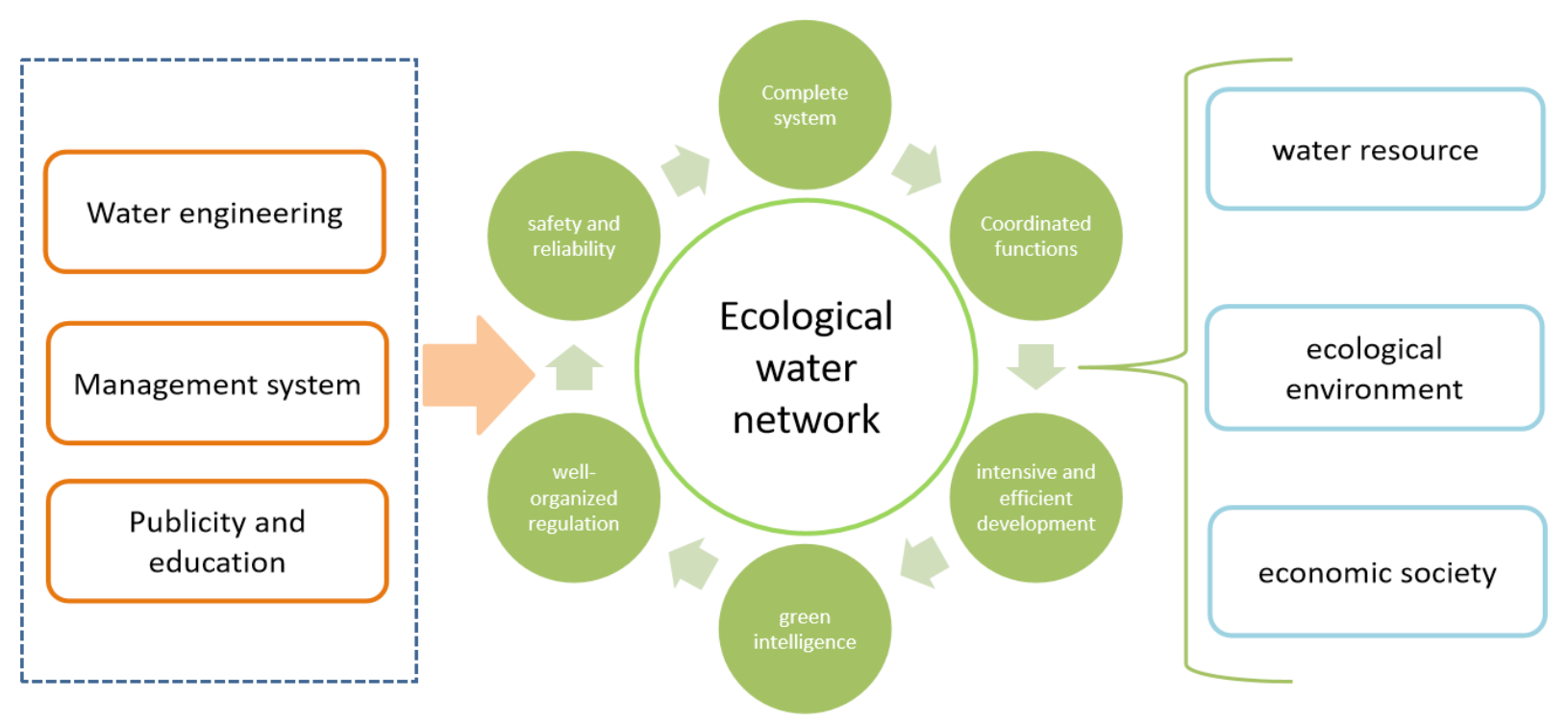

Figure 1. overall development idea of ecological water network

department need to strengthen the construction of different size water conservancy infrastructure and ecological corridor by learning from the effective investment and financing means of various regions. Gradually improve Guangxi water conservancy facilities network by integrating water diversion, water storage, water distribution, water supply and energy conservation. The government should lay emphasis on the construction of water source project, water conveyance channel and green ecological engineering with accelerating the development of ecological engineering industry. A regional data collection platform should be built by combining the digital watershed established by remote sensing satellite, UAV and other technical means with the existing monitoring stations to carry out real-time online supervision of all elements of basic data such as ecology, environment and hydrology. The optimal scheduling of ecological water network is expected to complete through data-driven, supply and demand analysis. Finally, the beautiful Guangxi with clear water and harmony between people and water will be achieved.

\subsection{Comprehensive Management of Water Ecological Environment}

The report of the 19th National Congress of the Communist Party of China ${ }^{[26]}$ proposed "optimizing the ecological security barrier system, building ecological corridor and biodiversity protection network". We should promote and implement the ecological protection and restoration projects of rivers and lakes, groundwater protection and restoration projects and the construction projects of ecological environment outside the river as soon as possible. Moreover, we should control the over exploitation and utilization of water ecology by human economic and social activities, explicitly prohibit fishing, hunting, illegal sand mining and illegal construction within the red line of the nature reserve, clarify the law enforcement power of the administrative agencies of the nature reserve, and improve the law enforcement ability of the law enforcement team. To ensure that the ecological water network has a complete ecological function.

\subsection{Improve the Management System of Ecological Water Network}

By improving the legal system, reforming the system, and improving the mechanism ${ }^{[27]}$, we should implement the regulatory responsibility, and establish a comprehensive, efficient, and clear division of labor supervision system for the ecological water network system, by which the regulatory work has laws to follow and rules to follow. From the construction of ecological water network infrastructure to the post project operation and maintenance, the responsible departments and personnel should be clearly implemented to ensure the smooth operation of ecological water network and the comprehensive and stable monitoring data, as well as the perfect regional eco-environmental water security system. According to the development and utilization conditions of regional water resources and the water demand of ecological environment, the control index of water resources allocation and allocation should be clarified and the construction of dispatching operation and management system platform should be strengthened to promote the unified allocation of basin and regional water resources. 


\subsection{Strengthen the Propaganda and Education of Water Ecological Protection}

Through cooperation with local colleges and social organizations, we encourage the concept of water ecological protection to enter the classroom to improve the public's awareness of water ecological protection, and strengthen the training and education of water ecological protection management personnel, moreover, elaborately build water ecological park to make the tourists experience the natural customs of human water harmony, to publicize the important value of ecological protection to the whole society through Internet and we media, in addition, vigorously publicize the relevant administrative regulations and management regulations to improve the public's sense of responsibility for the supervision of the ecological environment with forming a public opinion atmosphere that attaches importance to the protection of water sources and the protection of the ecological environment in the whole region.

\section{Conclusions}

The construction of ecological water network is a stepby-step system process. The ultimate goal is to establish a systematic ecological water network with complete system, coordinated functions, well-organized regulation, safety and reliability. In summary, firstly, enhance the construction of ecological water network, and realize the clear water intercommunication and data management platform construction of ecological water network. The second is to put an end to the destruction of the ecological environment by speeding up the work of ecological environment protection and governance and strengthening the law enforcement of management. The third is to perfect the relevant laws and regulations. The departments should implement the regulatory responsibility and security system. The fourth is to make greater efforts to publicize and expand social impact, meaning that enhance the awareness of the whole people to participate. By which the efficient and healthy development of ecological water network in Guangxi will be realized ultimately.

\section{References}

[1] Huang Guoqin, Wang Shubin, Zhao Qiguo. Ecological agriculture in Guangxi: course, effect, problems and countermeasures [J]. Acta ecologica Sinica, 2014, 34 (18): 5153-5153.

[2] Fang Zijie, Xia Yuli, Tang Yanbiao. Exploration and Thinking on building a "happy big water network" in Zhejiang in the new era $[\mathrm{J}]$. China water conservancy, 2020, no.890 (08): 27-30+33.
[3] Liu Yongyi, Wang Weiping, fan Mingyuan, et al. Study on the strategy of modern water network construction and optimal allocation of water resources in Shandong Province $[\mathrm{C}] \quad$ the eighth excellent academic papers of Shandong Water Conservancy Society.

[4] Li Guoying. Practice and effect of "Digital Yellow River" project construction [J]. China water conservancy, 2008 (07): 38-40.

[5] Tian Jiangtao, Liu Jiping. Design and implementation of Digital Qingjiang GIS platform [J]. Guangxi water conservancy and hydropower, 2003 (01): 1517.

[6] Ren Liliang, Liu Xinren, Renli Liang, et al. Research progress of digital elevation model information extraction and digital hydrological model [J]. Advances in water science, 2000, 11 (4): 463-469.

[7] Yi Ming. Intelligent water network, a comprehensive carrier of water conservancy modernization [J]. Research on water conservancy development, 2013 (3): $1-5$.

[8] Kuang Shangfu, Wang Jianhua. Building a national intelligent water network project to enhance China's water security capability $[\mathrm{J}]$. China water conservancy, 2013 (19): 27-31.

[9] Chen Chen. Research and application of water resources allocation based on digital water network in the receiving area of water diversion from Hanjiang River to Weihe River [D]. Xi' an University of technology, 2018.

[10] Xie T F , Beijing, China. Optimal allocation of water resources in relation to eco-environmental water demand. Water Resources and Hydropower Engineering, 2004.

[11] Li Jin, Jin Xiaobin, sun Rui, Gu Zhengming, Han Bo, Zhou Yinkang. Preliminary study on the construction of the regional composite ecological network of Jiangnan Water Network -- Taking Jintan District of Changzhou City as an example $[\mathrm{J}]$. Resources and environment of the Yangtze River Basin, 2020, V.29 (11): 95-103.

[12] Hu D . Water resources law and protection of eco-environmental water provision in UK[J]. China Water Resources, 2010.

[13] Jiang Tibing, Huang Tianrong, Liu Jinxiu. Wuhan City, Hubei Province: building Jiangcheng ecological water network and building a harmonious city of human and water [J]. China water conservancy, 2011 (1): 68-68.

[14] Fath B D , Scharler U M , Ulanowicz R E , et al. Ecological network analysis: network construc- 
tion[J]. Ecological Modelling, 2017, 208(1):49-55.

[15] Tu Qiang, Chen Qiyuan, Lu Li. Strengthening the construction and supervision of small and medium hydropower development in Guangxi Zhuang Autonomous Region [J]. Science and technology guide, 2009, 027 (011): 18.

[16] Li Hongliang, Yan Qinghong, Huang He, et al. Current situation and Development Countermeasures of water conservancy landscape resources in Guangxi [J]. China water conservancy, 2016 (1): 28-31.

[17] Zhuang Wanying. Investigation and evaluation of water conservancy landscape resources in Guangxi $[\mathrm{J}]$. Guangxi water conservancy and hydropower, 2017, 000 (004): 86-89.

[18] Xie Yuchu, Zhang Suxin, Lin Bing, et al. Spatial division of land ecological restoration in Guangxi county based on supply and demand of ecosystem services [J]. Journal of natural resources, 2020.

[19] He Yun. Occurrence regularity of forest fire in Guangxi and its correlation with meteorological factors [D]. Central South University of forestry science and technology, 2018.

[20] Lin kunyong. Promoting the construction of beautiful Guangxi under the guidance of ecological civilization [J]. City, 2017 (5).

[21] Huang Cao, CHEN Ye Hua, Li Zhi Wei, et al. Optimization of water system pattern and connectivity in Dongting Lake area $[\mathrm{J}]$. Advances in water science,
2019, V. 30; No.152(05):51-62.

[22] Wu Weixing, Xiong Xiaowen. Analysis on the 13th five year plan of Guangxi water conservancy development $[\mathrm{J}]$. Guangxi water conservancy and hydropower, 2015, 02 (2): 15-15.

[23] Zheng Jiangli, Li xingpin, fan Qunfang. Theoretical study on the rational allocation of water resources in the economic leapfrog development areas of southern China [J]. People's Pearl River, 2010, 31 (006): 2529.

[24] Wu Ling, you Yi Lai, Wang Xin, et al. Study on Countermeasures of constructing Zhuhai wetland ecological protection system and accelerating the construction of green ecological water network [J]. Environmental ecology, 2020, 002 (002): 93-97.

[25] Jiang Tiebing, Huang Tianrong, Liu Jinxiu. Wuhan City, Hubei Province: building Jiangcheng ecological water network, building a harmonious city of human and water $[\mathrm{J}]$. China water conservancy, 2011 (1): 68-68

[26] Fang Xuejuan, Ren Haibo, Wu Jinchao, et al. Research progress and Enlightenment of coastal landscape ecological pattern $[\mathrm{J}]$. Scientific and technological innovation and productivity, 2019, 000 (011): p.33-37.

[27] Guo xuning, He Jun, Zhang Haibin, et al. Some considerations on the construction of national water network system [J]. China water conservancy, 2019 (15). 